The Pediatric Infectious Disease Journal Publish Ahead of Print

DOI: 10.1097/INF.0000000000002212

\title{
Clinical Characteristics of Infective Endocarditis in Children
}

Jelte Kelchtermans, MD (1), Lorenz Grossar, MD (1), Benedicte Eyskens, MD, PhD (1), Bjorn Cools, MD (1), Mieke Roggen, MD (1), Derize Boshoff, MD, PhD (1), Jacoba Louw, MD, PhD (2), Stefan Frerich, MD, PhD (2), Tiago Rafael Veloso, PhD (1), Jorien Claes, PhD (1), Bartosz Ditkowski, PhD (1), Filip Rega, MD, PhD (3), Bart Meyns, MD, PhD (3), Marc Gewillig, MD, $\mathrm{PhD}$ (1), and Ruth Heying, MD, PhD (1)

Pediatric Cardiology, Department of Cardiovascular Developmental Biology, KU Leuven, Belgium (1), Pediatric Cardiology, AZM Maastricht, The Netherlands (2), Division of Clinical Cardiac Surgery, Department of Cardiovascular Sciences, KU Leuven, Leuven, Belgium (3) Corresponding author: Ruth Heying, Department of Pediatric Cardiology, UZ Leuven Herestraat 49, 3000 Leuven, Belgium . Phone: +32 16 343865; Fax: +32 16 343981; Email: ruth.heying@uzleuven.be Abbreviated Title: Characteristics of Infective Endocarditis in Children

Running Head Infective Endocarditis in Children

Conflicts of Interest and Source of Funding:

This study was sponsored by a grant of the Research Fund KU Leuven (OT/14/097) given to RH. TRV was sponsored as a Postdoctoral Fellow of the Research Foundation Flanders (FWO Grant Number - 12K0916N) and RH by the Clinical Research Fund of UZ Leuven. For the other authors none were declared. 


\section{ABSTRACT}

Background: Infective endocarditis (IE) remains a diagnostic and therapeutic challenge associated with high morbidity and mortality. We evaluated the microbial profile and clinical manifestation of IE in children.

Methods: A retrospective study examining pediatric IE cases treated between 2000 and 2017 at the Department of Pediatric Cardiology, KU Leuven, was conducted. Clinical presentation, treatment, complications, outcome of IE, underlying microorganisms and congenital heart defects were reviewed.

Results: 53 patients were diagnosed with IE. Overall, 19 patients (36\%) required cardiac surgery. 7 patients (13\%) died. $87 \%$ of patients had an underlying congenital cardiac defect. 18 $(34 \%)$ children presented with prosthetic graft IE. A causative organism was found in $49(92 \%)$ cases: viridans group streptococci were identified in 17 (32\%), S. aureus in $13(25 \%)$ and coagulase-negative staphylococci (CNS) in 11 (20\%) children. Community acquired (CA) IE increased significantly from $8(33 \%)$ cases in $2000-2007$ to $20(74 \%)$ cases in $2008-2017$ $(p<0.01)$. Even with viridans streptococci being significantly more prevalent in the CA group $(p<0.01)$, we did not observe an increase of streptococcal IE from 2008 - 2017. 17 (32\%) patients presented with HA IE during the first year of life with $14(82 \%)$ children after surgery and a prevalence of CNS (53\%).

Conclusion: The incidence of pediatric IE was similar over the investigated time period with a shift towards CA IE. Streptococci and staphylococci accounted for the majority of cases in both periods. Awareness of IE and its prevention is crucial in patients after implantation of prosthetic grafts.

Keywords: congenital heart disease, infective endocarditis, artificial valve conduits 


\section{INTRODUCTION}

Patients with congenital and acquired heart valve disease are at higher risk to develop infective endocarditis (IE). In clinical practice, IE has proven itself a challenging diagnosis with high morbidity and mortality (1-4). Novel therapeutic strategies in congenital heart disease (CHD), including catheter- and surgery derived interventions, have improved patients quality of life but treatment associated complications, such as IE, are still of major concern (5-8).

Furthermore, advances in life saving medical interventions such as critical care and immunosuppressive therapies have increased the population at risk for IE and more frequent use of implanted prosthetic material leads to a higher incidence of device related infections (9-11). In children, patients with underlying CHD are the most important population at risk for IE $(12,13)$ and due to advanced treatment age at IE diagnoses decreases $(3,8,14)$.

Changes in IE epidemiology have become of major interest after the recommended IE prophylaxis has been restricted to patients at high risk from 2007 onwards $(15,16)$. Streptococci are still described as the most relevant bacterial pathogen responsible for IE $(8,17,18)$, whereas Staphylococcus aureus infections are becoming more frequent (19).

We conducted a retrospective investigation of pediatric patients with IE treated between 2000 and 2017 at our institution. By evaluating epidemiologic and clinical data with time we aimed to improve our understanding of the risk of IE associated with specific CHD, the course of disease and its potential prevention.

\section{PATIENTS AND METHODS}

\section{Patients:}

This retrospective study was conducted using the patient database of the Department of Pediatric Cardiology, University Hospital Leuven, a tertiary referral hospital. All children under the age 
of 16 years who were diagnosed with definite IE following the modified Duke Criteria between January, 1st 2000 and December, 31st 2017 were included in the study (20). Approval by the local ethics committee was asked and informed consent was taken from the parents.

\section{Clinical data:}

Patients' date of birth, gender, underlying CHD and surgical and interventional treatments were noted. Recorded data of the IE episode included date of IE diagnosis, patient's history, clinical presentation, echocardiographic findings, biochemical and microbiologic data, medical and surgical treatment, length of hospitalization, complications and outcome. Organisms were considered causal if at least two blood cultures or a single culture of intraoperative specimens were positive. If no organism met these criteria the IE episode was considered culture negative. Community acquired IE was defined as episodes occurring more than 6 months after cardiac surgery in non-hospitalized patients or within the first $72 \mathrm{~h}$ of hospitalization.

\section{Statistical analysis:}

Continuous variables are reported as mean \pm standard deviation. Categorical variables are mentioned as frequencies and percentages of the specific group. Statistical analysis was performed by application of the Fischer's and Chi-square test using GraphPad Prism (7.0d; GraphPad Software, San Diego California USA).

\section{RESULTS:}

\section{Population characteristics:}

Analysis of patient records identified 53 children diagnosed with definite IE according to the Modified Duke Criteria under the age of 16 between January, 1st 2000 and December, 31st 2017. None of the patients had recurrent episodes of IE. 
Our patient population consisted of $34(64 \%)$ boys and $19(36 \%)$ girls with a mean age at diagnosis of 6.5 years. We observed 17 infants under the age of 1 year with 11 of them diagnosed between 2000 and 2007. Overall, 24 patients were diagnosed between 2000 and 2007 while 29 patients were diagnosed from 2008 to 2017. In the entire period 7 patients (13\%) died from IE and 19 patients (36\%) required cardiac surgery in the management of IE. All patients were treated for 6 weeks with the respective recommended antibiotic treatment according to the ESC guidelines (21).

Characteristics of IE patients are shown in Table 1 and patients' age distribution is given in Figure 1.

\section{Underlying congenital heart disease}

46 of the 53 patients (87\%) had underlying CHD which included mainly Tetralogy of Fallot (TOF) in 13 patients, a perimembranous yentricular septal defect (pmVSD) in 6 patients and a truncus arteriosus in 4 patients. None of the patients had an underlying muscular VSD or atrial septal defect (ASD) and none rheumatic disease. At the time of IE diagnoses, 8 (17\%) patients with CHD had not yet undergone surgical repair for their lesion. Detailed diagnoses of CHD are shown in Table 2. In our study population $7(13 \%)$ children had a structural normal heart with 2 of them having undergone immunosuppressive treatment.

\section{Microbiology:}

A causative organism was found in 49 cases (92\%). In 6 patients, 2 different causative organisms were withheld and included in the analysis. The most frequent causative organisms in the total study period from 2000 - 2017 were viridans group streptococci (17 episodes, 29\%), S. aureus (13 episodes, 22\%) and coagulase-negative staphylococci (11 episodes, 19\%). Other organisms included Candida, enterococci, members of the HACEK group and Abiotrophia species, as well 
as Mycobacterium avium and Serratia marcescens. Patients were divided into two groups depending on the time point of IE diagnosis: 2000-2007 for group 1 and 2008-2017 for group 2. There was no significant difference in the prevalence of any underlying organism, also not for viridans group streptococci comparing the 2 time periods $(p>0.05)$. Figure 2 demonstrates the relative importance of the underlying organisms in both groups.

Using the provided criteria, 28 of all cases were classified as community acquired (CA) IE while 25 cases were classified as hospital acquired (HA) IE. Interestingly, CA IE cases were significantly more frequent between 2008 and 2017 compared to the earlier period $(p<0.01$, Table 1 and Figure 3A).

In general, viridans group streptococci are the major underlying cause for CA IE since they were found in $14(48 \%)$ of CA IE cases while only present in $3(10 \%)$ of HA IE cases $(p<0.01)$. Conversely, coagulase-negative staphylococci were present in 2 (7\%) of CA IE cases and 9 $(31 \%)$ of HA IE cases $(p<0.05)$. S. aureus accounts as the second important agent in CA IE 8 (27\%) cases compared to $5(17 \%)$ in HA IE. None of the patients was MRSA positive. Despite increasing CA IE in the later time period, the incidence of viridans streptococcal IE did not increase in CA IE between 2008-2017 ( $p>0.05$ ). Mycobacterium avium and Serratia marcescens caused HA IE. Figure 3B summarizes the relation of the three main causative organisms in the HA and CA group between 2000 - 2017.

\section{IE in children under 1 year of age:}

Age distribution among our patients is spread in a similar manner, besides a subpopulation of 17 patients $(32 \%)$ in which IE was diagnosed during the first year of life. All patients, even younger than five months, presented with HA IE. 16 of them had underlying CHD and in 14 patients IE was diagnosed 30 days ( \pm 42 days) after cardiac surgery. Looking at the causative organisms 
within this subgroup revealed that coagulase-negative staphylococci were much more prevalent (53\% against $9 \%$ at older age, $p<0.05$ ) while viridans group streptococci were not present. In addition, S. aureus was found in 3 children and all 4 children with Candida IE belonged to the subgroup of postoperative infants.

\section{Lesion location:}

Analyzing cardiac ultrasound records, IE was classified as right sided IE in $33(62 \%)$ patients and left sided IE in 15 (28\%) patients. Five (10\%) patients presented with bilateral lesions (Table 2). Right heart IE was equally caused by the main 3 bacteria (S. aureus 10 episodes, coagulasenegative staphylococci 9 episodes and viridans group streptococci 12 episodes). There was also no prominent causal agent in left or bilateral heart IE. However, culture negative IE was observed in both, left and right heart IE.

All 18 patients with underlying TOF or truncus arteriosus had undergone previous surgery and presented with right-sided IE lesions which were associated with left-sides lesions in 3 patients. The majority of this sub-group (15 patients, $83 \%$ ) presented with infection of prosthetic material. The primary IE site was determined to be a pulmonary homograft in 6 patients, a stent mounted bovine jugular vein (BJV) graft in 4 patients, a BJV graft in 3 and a bovine pericardial patch (RVOT) in 2 children. The remaining 3 patients (all TOF) had native tricuspid valve IE and 1 patient had both native mitral and pulmonary valve involvement.

In total, 18 of the 53 patients (34\%) presented with IE of a prosthetic valve conduit. None of the children had an artificial heart valve in place.

Out of the 7 children with a structural normal heart 4 patients had a right sided IE (tricuspid valve) and 3 children a left sided IE (mitral valve). A CT heart / thorax was not required for IE 
localization but has been performed to visualize associated complications such as long emboli in 8 patients and an aortic aneurysm in 1 patient. Table 2 shows the localization of IE in all patients.

\section{Embolic risk and surgical treatment:}

Six out of $33(18 \%)$ children diagnosed with right heart IE presented with long emboli visualized by CT not leading to respiratory impairment. Left heart IE led to peripheral emboli in 5 patients and 1 patient presented with a central embolization causing hemiplegia. Embolic complications were not observed in patients with bilateral IE lesions. One patient presented with an aortic root and cerebral abscess.

Nineteen out of $53(36 \%)$ children required surgical treatment of IE with 6 patients undergoing urgent surgery at time of IE diagnosis and 6 patients at 7-15 days after IE diagnosis. In followup, additional 7 patients underwent late surgery after 1-9 months. Twelve children were operated for right heart IE, 6 children for left heart IE and one for bilateral IE. Among these patients we observed 5 patients with tricuspid valve involvement, 5 patients with mitral valve involvement and 2 with aortic valve involvement. Interestingly, 8 patients with right heart IE needed operative treatment for IE of a prosthetic graft or patch (4 stent mounted BJV grafts, 2 BJV grafts, 1 bovine patch, 1 Gore-Tex conduit).

S. aureus, viridans group streptococci and S. epidermidis were the prominent underlying microorganisms in patients who required surgical treatment (both staphylococci 5 patients, streptococci in 6 patients). All patients but one had an underlying CHD. In total, 23 (43\%) children required intensive care treatment including $8(15 \%)$ patients under mechanical ventilation. 


\section{Clinical features at IE diagnosis:}

Patients' record analysis enabled detailed assessment of presenting complaints in $35(66 \%)$ cases. Despite a thorough review, in several patients it was unclearly documented what initial symptoms led the primary team to consider IE as a possible diagnosis before admission. We therefore did not include these patients in the analysis of clinical features at IE presentation. The majority of patients presented initially with fever of unknown origin (20 of these cases (57\%)). A further $9(25 \%)$ cases presented with sepsis. 3 (9\%) patients presented with septic embolisms, 2 to the hip, and the other to the left foot. An abscess at the sternotomy, dyspnea, toxic shock syndrome and joint complaints in combination with a new cardiac murmur was present each in one patient.

\section{Outcome:}

Overall mortality was $13 \%$ including one 14 -year-old patient with CA IE caused by S. aureus that passed away 6 months after medical and surgical treatment of IE due to heart failure. The remaining 6 in-hospital mortalities occurred in HA IE during the first year of life between 2000 and 2004. In these 7 cases, 4 causal organisms were found: coagulase-negative staphylococci present in 4 cases and S. aureus, Candida albicans and Enterococcus faecalis each present in 1 case.

\section{DISCUSSION}

Analyzing clinical and microbial characteristics of children diagnosed with definite IE, our data clearly indicate the relevance of underlying CHD as a major predisposing risk factor of IE. In addition, a need of surgical intervention in $36 \%$ of our population and a mortality rate of $13 \%$ indicates that IE is still associated with relevant morbidity and mortality. 
Since an underlying CHD was known in $87 \%$ of our IE population data strongly support the observation of other reports which state that CHD became one of the major risk factors for IE after the occurrence of rheumatic fever declined $(9,14,22,23)$. In addition we observed a high prevalence (62\%) of right-sided IE in a CHD heavy population as observed in other CHD cohorts $(8,22,24)$.

Interestingly, IE in unrepaired CHD was less common in our patients. 4 out of 53 patients had an unrepaired pmVSD, partially associated with valve anomalies or a coarctation. Remarkably, we found no IE in patients with ASDs and only 1 episode due to a patent arterial duct. Although patients with an univentricular physiology or cyanosis in general are known to be at higher risk to develop IE, we did only recognize 5 out of 53 patients (15). In contrast, patients after TOF or truncus arteriosus repair were very prevalent in our population (18 patients). Due to the improvement in survival of patients with complex CHD more patients appear after surgical treatment with shunts or implanted devices, developing an increasing risk population at pediatric and adult age $(3,9)$. Our data verify this evolution by seeing IE in the majority of patients after corrective heart surgery and in $34 \%$ after implantation of a prosthetic valve conduit including the cryopreserved homograft and bovine jugular vein grafts. Sample size of our patient cohort does not allow any suggestion on susceptibility for bacterial adhesion to the different valve conduits, but recent clinical observations give special awareness to IE as a complication in patients having undergone transcatheter pulmonary valve replacement using BJV conduits $(25,26)$. Larger, multicentre studies are desirable to focus on IE in patients with implanted prosthetic material to gain knowledge on IE pathogenesis in this context in which pathways evoking inflammation and fibrin deposition are not fully understood yet. 
It has been stated that the right heart in general is more susceptible for device related infections, probably due to hemodynamic factors $(8,27)$. Cardiac malformations differ in their specific substrate for IE with various disturbances in flow patterns (8). A non-physiologic flow pattern can favor bacterial adhesion on native or prosthetic valves and, on the other hand, endothelial damage can result of abnormal turbulent blood flow, both mechanisms being considered an IE inciting event $(1,28)$.

IE in children with a normal structured heart remains present in a small group of our population (13\%). At present, $8-20 \%$ of the IE cases are estimated to occur in children with anatomically normal hearts, in which the course of IE is essentially different if predisposing factors as indwelling central lines leading to health-care associated IE are present $(24,29)$. Relatively absent in our patient population were immunocompromised patients ( 2 children). This supports the hypothesis that endothelial damage associated with transient bacteremia plays a more dominant role in IE pathogenesis than the immunocompromised state (1).

Focusing at age at IE diagnosis, a group that stands out in our dataset is the large group of patients under 1 year of age. An elevated risk of IE in infants with CHD is known and even described in up to $42 \%$ of the investigated pediatric IE population $(14,30)$. Knowing that operative treatment for CHD is mainly performed in the first year of life, that and other reasons for intensive care treatment as well as central venous lines may contribute to put these infants at high risk for IE (31). Our study verifies previous cardiac surgery as being a risk factor in this age group as 14 out of 17 infants developed IE in the postoperative period. This was also associated with a high risk of adverse outcome and mortality, as previously reported $(15,32)$. IE caused by viridans group streptococci was the most prevalent in our population, closely followed by coagulase-negative staphylococci and S. aureus. This is in line with an Italian 
pediatric IE survey from 2000-2015 where streptococci were found as the major underlying cause in CHD patients (24). In other studies and an international review of more than 30,000 children, however, S. aureus is reported as being the most frequent and important bacterial origin $(19,22,32)$.

Interest has been raised on streptococcal IE after restriction of the antibiotic IE prophylaxis to only patients at high risk. Epidemiologic analysis of streptococcal IE showed no change in incidence before and after introduction of the restricted IE prophylaxis in 1157 US children with definite IE (33) while an increase was seen only for the pediatric age group between 10-17 years in a more recent study (34). As well as in our study, unfortunately no conclusive information on antibiotic IE prophylaxis was available. In our complete study population we saw an unchanged incidence of IE caused by viridans streptococci over the whole time period ( $29 \%$ vs $35 \%$ in the later time interval), nevertheless, statistical power being limited by the relatively small patient number.

Furthermore, our data indicate a shift from HA IE towards CA IE with a significant increase of CA IE in 2008-2017 (69\%). As mentioned, even though streptococci were the most frequent organisms in CA IE, overall incidence of streptococcal IE remained the same.

Conversely, a higher prevalence of HA IE was found in pediatric IE cohorts from New Zealand and USA $(23,35)$. The divergence in evolution could be caused by the different observational periods or geographic region. In contrast to HA IE the microbiological pattern of CA IE seems relatively consistent across the globe with streptococcal species being the most prevalent (3537).

In contrast, in patients diagnosed before 1 year of age no IE caused by viridans streptococci was seen and most cases were caused by coagulase-negative staphylococci, fungi or S. aureus. This 
microbiological pattern seems consistent with earlier findings in neonates and young infants (14, 31) potentially correlating with an increased susceptibility and exposure to these microorganisms related to frequent HA IE. In our population, fungi and enterococci were rare and exclusively found in this specific subpopulation, having chronic indwelling catheters (15). Culture negative IE remains present in a minority of our children with a decreasing general trend as microbiological diagnostics have improved $(4,15)$.

The classic clinical hallmarks of IE, such as fever and sepsis, remain major clinical signs found at presentation. This highlights the importance of a high index of suspicion, especially in patients with a history of CHD and implanted prosthetic material $(3,4,38)$.

Outcome is influenced by many factors as the virulence of the underlying microorganism and the requirement of surgery (37). The need of surgical treatment in $36 \%$ of our patient cohort is similar to that of other series and reflects the high morbidity due to IE $(4,39)$. The most frequent performed operation was a valved graft replacement in the right ventricular outflow tract. Surgical treatment was curative in all but 1 patient. We did not observe an associated mortality with the need of surgical treatment.

Our overall mortality is comparable with results from recent pediatric IE series describing a mortality rate of about $10 \%(8,9)$. Mortality was concentrated in those children diagnosed $<1$ year of age and before 2004. The sharp decline after 2004 highlights the potential impact of vigilance and early treatment, especially in a high-risk population.

Prevalence and prevention of IE gained attention in the context of changed guidelines for IE prophylaxis from 2007 onwards $(21,40)$. As also shown by our data, the vast majority of patients presenting with IE are still included in the classification of high risk patients and therefore eligible for antibiotic IE prophylaxis. Given the limits of antibiotic prophylaxis and available 
therapeutic interventions, it is likely that further improvement in IE outcome has to focus on different prevention measures, as also included in the 2015 actualized ESC IE guidelines (21, 41). Studies evaluating the knowledge on IE in CHD patients or parents show a striking lack of understanding IE, dental hygiene and awareness of symptoms requiring an adequate medical investigation and no self-treatment (42-44). Therefore, a structured education of physicians and patients seems to be a necessary major target in daily practice to improve knowledge and thereby contributing to a decrease in diagnostic delay of IE, which still represents an important burden of the disease. 


\section{REFERENCES}

1. Moreillon P, Que YA. Infective endocarditis. Lancet. 2004;363:139-149.

2. Holland TL, Baddour LM, Bayer AS, Hoen B, Miro JM, Fowler VG, Jr. Infective endocarditis. Nature reviews Disease primers. 2016;2:16059.

3. Di Filippo S, Delahaye F, Semiond B, et al. Current patterns of infective endocarditis in congenital heart disease. Heart. 2006;92:1490-1495.

4. Dixon G, Christov G. Infective endocarditis in children: an update. Curr Opin Infect Dis. 2017;30:257-267.

5. Hill EE, Herijgers P, Claus P, Vanderschueren S, Peetermans WE, Herregods MC. Clinical and echocardiographic risk factors for embolism and mortality in infective endocarditis. Eur $J$ Clin Microbiol Infect Dis. 2008;27:1159-1164.

6. Van Dijck I, Budts W, Cools B, et al. Infective endocarditis of a transcatheter pulmonary valve in comparison with surgical implants. Heart. 2014.

7. Mery CM, Guzman-Pruneda FA, De Leon LE, et al. Risk factors for development of endocarditis and reintervention in patients undergoing right ventricle to pulmonary artery valved conduit placement. J Thorac Cardiovasc Surg. 2016;151:432-439, 441.e431-432.

8. Knirsch W, Nadal D. Infective endocarditis in congenital heart disease. Eur J Pediatr. $2011 ; 170: 1111-1127$.

9. Rosenthal LB, Feja KN, Levasseur SM, Alba LR, Gersony W, Saiman L. The changing epidemiology of pediatric endocarditis at a children's hospital over seven decades. Pediatr Cardiol. 2010;31:813-820.

10. Cooper DS, Jacobs JP, Moore L, et al. Cardiac extracorporeal life support: state of the art in 2007. Cardiol Young. 2007;17 Suppl 2:104-115. 
11. Weber R, Berger C, Balmer C, et al. Interventions using foreign material to treat congenital heart disease in children increase the risk for infective endocarditis. Pediatr Infect Dis J. 2008;27:544-550.

12. Johnson JA, Boyce TG, Cetta F, Steckelberg JM, Johnson JN. Infective endocarditis in the pediatric patient: a 60-year single-institution review. Mayo Clin Proc. 2012;87:629-635.

13. Coward K, Tucker N, Darville T. Infective endocarditis in Arkansan children from 1990 through 2002. Pediatr Infect Dis J. 2003;22:1048-1052.

14. Rushani D, Kaufman JS, Ionescu-Ittu R, et al. Infective endocarditis in children with congenital heart disease: cumulative incidence and predictors. Circulation. 2013;128:1412-1419. 15. Elder RW, Baltimore RS. The Changing Epidemiology of Pediatric Endocarditis. Infect Dis Clin North Am. 2015;29:513-524.

16. Bates KE, Hall M, Shah SS, Hill KD, Pasquali SK. Trends in infective endocarditis hospitalisations at United States children's hospitals from 2003 to 2014: impact of the 2007 American Heart Association antibiotic prophylaxis guidelines. Cardiol Young. 2016:1-5. 17. Que YA, Moreillon P. Infective endocarditis. Nat Rev Cardiol. 2011;8:322-336.

18. Baltimore RS, Gewitz M, Baddour LM, et al. Infective Endocarditis in Childhood: 2015 Update: A Scientific Statement From the American Heart Association. Circulation. $2015 ; 132: 1487-1515$.

19. Vogkou CT, Vlachogiannis NI, Palaiodimos L, Kousoulis AA. The causative agents in infective endocarditis: a systematic review comprising 33,214 cases. Eur J Clin Microbiol Infect Dis. 2016;35:1227-1245. 
20. Tissieres P, Gervaix A, Beghetti M, Jaeggi ET. Value and limitations of the von Reyn, Duke, and modified Duke criteria for the diagnosis of infective endocarditis in children. Pediatrics. 2003;112:e467.

21. Habib G, Lancellotti P, Antunes MJ, et al. 2015 ESC Guidelines for the management of infective endocarditis: The Task Force for the Management of Infective Endocarditis of the European Society of Cardiology (ESC). Endorsed by: European Association for Cardio-Thoracic Surgery (EACTS), the European Association of Nuclear Medicine (EANM). Eur Heart J. 2015;36:3075-3128.

22. Wang W, Sun H, Lv T, Tian J. Retrospective studies on pediatric infective endocarditis over 40 years in a mid-west area of China. Cardiology. 2014;128:88-91.

23. Webb R, Voss L, Roberts S, Hornung T, Rumball E, Lennon D. Infective endocarditis in New Zealand children 1994-2012. Pediaty Infect Dis J. 2014;33:437-442.

24. Esposito S, Mayer A, Krzysztofiak A, et al. Infective Endocarditis in Children in Italy from 2000 to 2015. Expert review of anti-infective therapy. 2016;14:353-358.

25. Malekzadeh-Milani S, Ladouceur M, Patel M, et al. Incidence and predictors of Melody(R) valve endocarditis: a prospective study. Archives of cardiovascular diseases. 2015;108:97-106. 26. Patel M, Malekzadeh-Milani S, Ladouceur M, Iserin L, Boudjemline Y. Percutaneous pulmonary valve endocarditis: incidence, prevention and management. Archives of cardiovascular diseases. 2014;107:615-624.

27. Morris CD, Reller MD, Menashe VD. Thirty-year incidence of infective endocarditis after surgery for congenital heart defect. Jama. 1998;279:599-603. 
28. Knirsch W, Haas NA, Uhlemann F, Dietz K, Lange PE. Clinical course and complications of infective endocarditis in patients growing up with congenital heart disease. Int J Cardiol. 2005;101:285-291.

29. Marom D, Ashkenazi S, Samra Z, Birk E. Infective endocarditis in previously healthy children with structurally normal hearts. Pediatr Cardiol. 2013;34:1415-1421.

30. Sun LC, Lai CC, Wang CY, et al. Risk factors for infective endocarditis in children with congenital heart diseases - A nationwide population-based case control study. Int I Cardiol. 2017;248:126-130.

31. Daher AH, Berkowitz FE. Infective endocarditis in neonates. Clinical pediatrics. 1995;34:198-206.

32. Day MD, Gauvreau K, Shulman S, Newburger JW. Characteristics of children hospitalized with infective endocarditis. Circulation. 2009;119:865-870.

33. Pasquali SK, He X, Mohamad Z, et al. Trends in endocarditis hospitalizations at US children's hospitals: impact of the 2007 American Heart Association Antibiotic Prophylaxis Guidelines. Am Heart J. 2012;163:894-899.

34. Sakai Bizmark R, Chang RR, Tsugawa Y, Zangwill KM, Kawachi I. Impact of AHA's 2007 guideline change on incidence of infective endocarditis in infants and children. Am Heart J. 2017; 189:110-119.

35. Tseng WC, Chiu SN, Shao PL, et al. Changing spectrum of infective endocarditis in children: a 30 years experiences from a tertiary care center in Taiwan. Pediatr Infect Dis J. 2014;33:467471.

36. Simsek-Yavuz S, Sensoy A, Kasikcioglu H, et al. Infective endocarditis in Turkey: aetiology, clinical features, and analysis of risk factors for mortality in 325 cases. International journal of 
infectious diseases : IJID : official publication of the International Society for Infectious Diseases. 2015;30:106-114.

37. Damasco PV, Ramos JN, Correal JC, et al. Infective endocarditis in Rio de Janeiro, Brazil: a 5-year experience at two teaching hospitals. Infection. 2014;42:835-842.

38. Murdoch DR, Corey GR, Hoen B, et al. Clinical presentation, etiology, and outcome of infective endocarditis in the 21st century: the International Collaboration on EndocarditisProspective Cohort Study. Arch Intern Med. 2009;169:463-473.

39. Tansel T, Onursal E, Eker R, Ertugrul T, Dayioglu E. Results of surgical treatment for infective endocarditis in children. Cardiol Young. 2005;15;621-626.

40. Wilson W, Taubert KA, Gewitz M, et al. Prevention of infective endocarditis: guidelines from the American Heart Association: a guideline from the American Heart Association Rheumatic Fever, Endocarditis, and Kawasaki Disease Committee, Council on Cardiovascular Disease in the Young, and the Council on Clinical Cardiology, Council on Cardiovascular Surgery and Anesthesia, and the Quality of Care and Outcomes Research Interdisciplinary Working Group. Circulation. 2007;116:1736-1754.

41. Toyoda N, Chikwe J, Itagaki S, Gelijns AC, Adams DH, Egorova NN. Trends in Infective Endocarditis in California and New York State, 1998-2013. Jama. 2017;317:1652-1660.

42. Van Deyk K, Pelgrims E, Troost E, et al. Adolescents' understanding of their congenital heart disease on transfer to adult-focused care. Am J Cardiol. 2010;106:1803-1807.

43. Knochelmann A, Geyer S, Grosser U. Maternal understanding of infective endocarditis after hospitalization: assessing the knowledge of mothers of children with congenital heart disease and the practical implications. Pediatr Cardiol. 2014;35:223-231. 
44. Bauer UMM, Helm PC, Diller GP, et al. Are adults with congenital heart disease informed about their risk for infective endocarditis and treated in accordance to current guidelines? Int $J$ Cardiol. 2017;245:105-108.

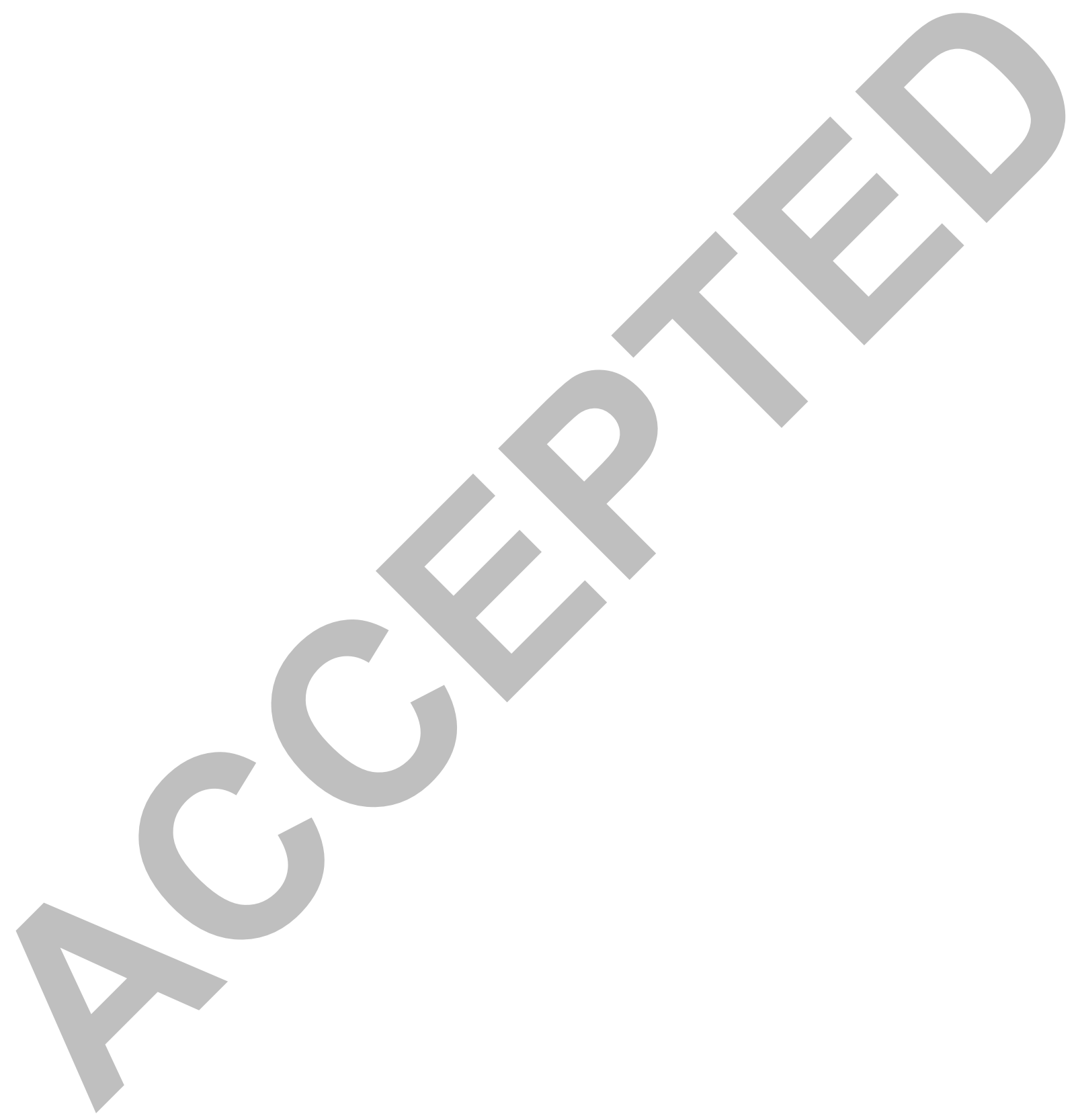




\section{LEGENDS}

\section{Figure 1. IE distribution upon age}

A similar distribution of age was found, besides a subpopulation with 17 children $<1$ year of age. IE $=$ infective endocarditis.

\section{Figure 2. IE underlying organisms}

S. aureus, viridans group streptococci and coagulase-negative staphylococci (CNS) are the three major organisms causing IE in our patient population. There is no significant difference comparing the two time periods. IE = infective endocarditis, streptococci $=$ viridans group streptococci, Serratia sp. = Serratia marcescens, sp. = species, $\mathrm{CN}=$ culture negative.

Figure 3. Relative importance of hospital- and community acquired IE over time and main underlying organisms

(A) Community acquired (CA) infective endocarditis (IE) increased significantly in 2008 - 2017.

(B) In CA IE viridans group streptococci were the major causative agent, whereas coagulasenegative staphylococci (CNS) were significantly more present in the hospital acquired (HA) IE group between 2000 - 2017, respectively. Results are given in \% of the respective CA or HA group. $\mathrm{CN}=$ culture negative. $* *=p<0.01, *=p<0.05$. 
Figure 1

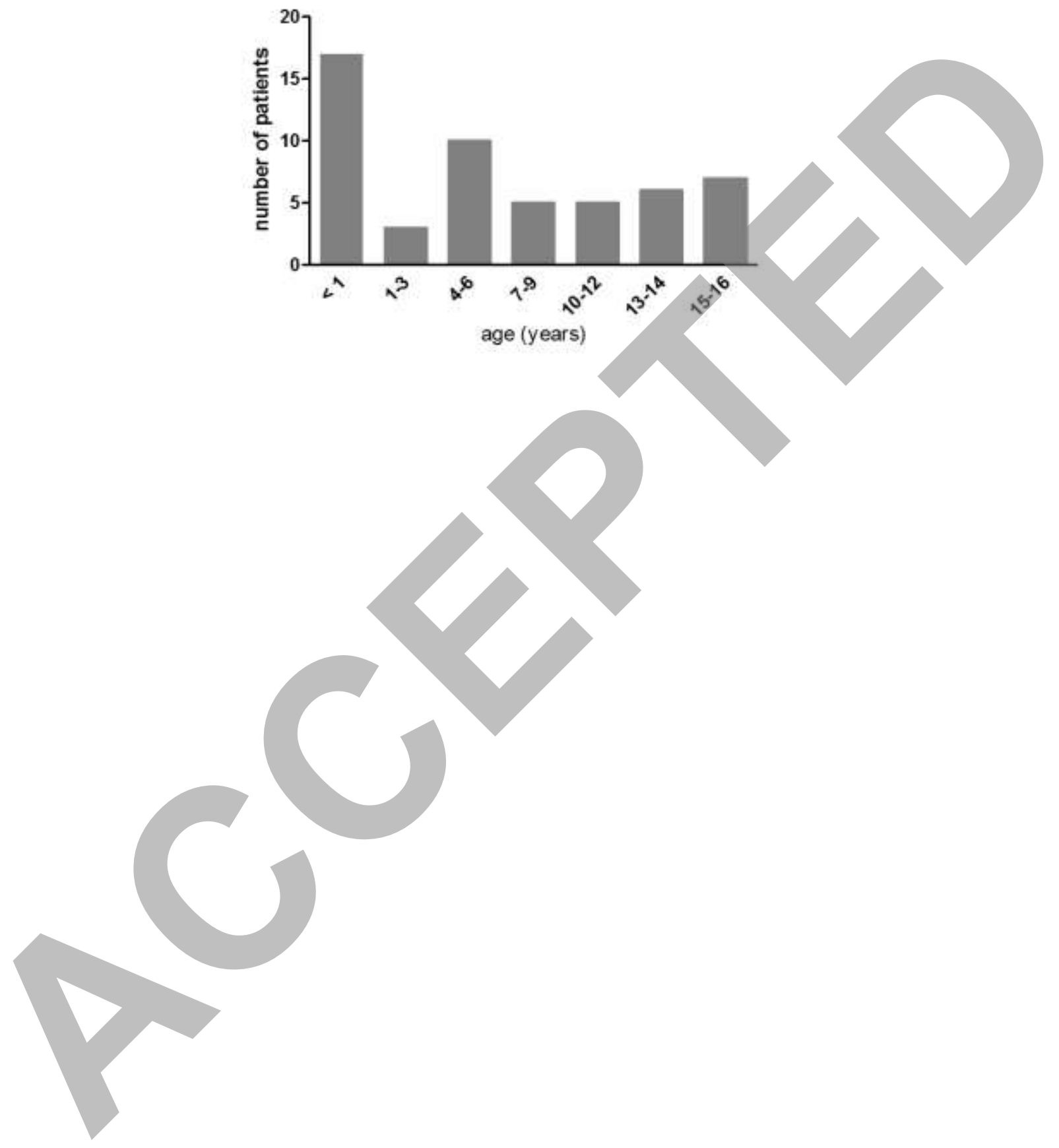


Figure 2

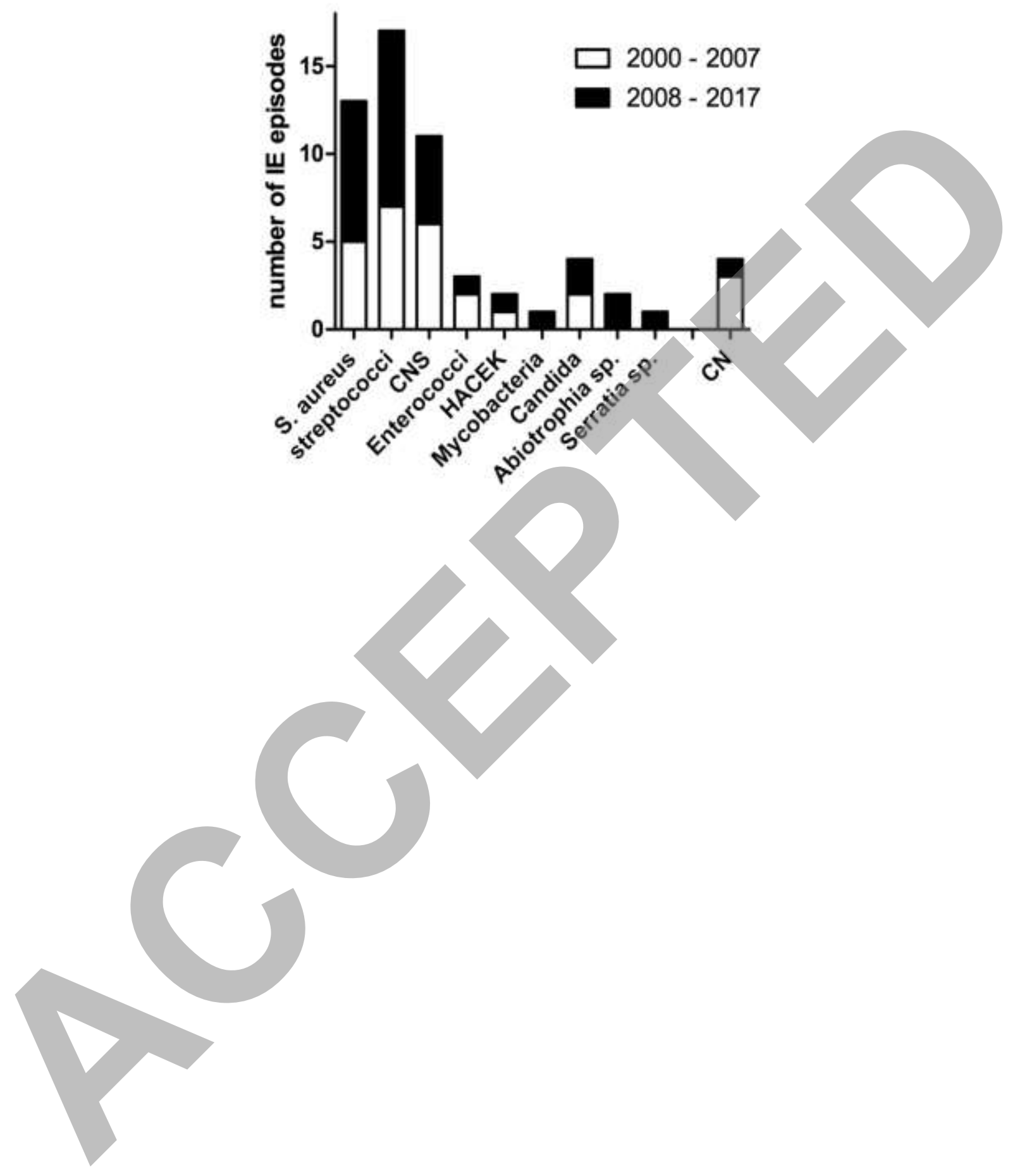


Figure 3

(A)

(B)

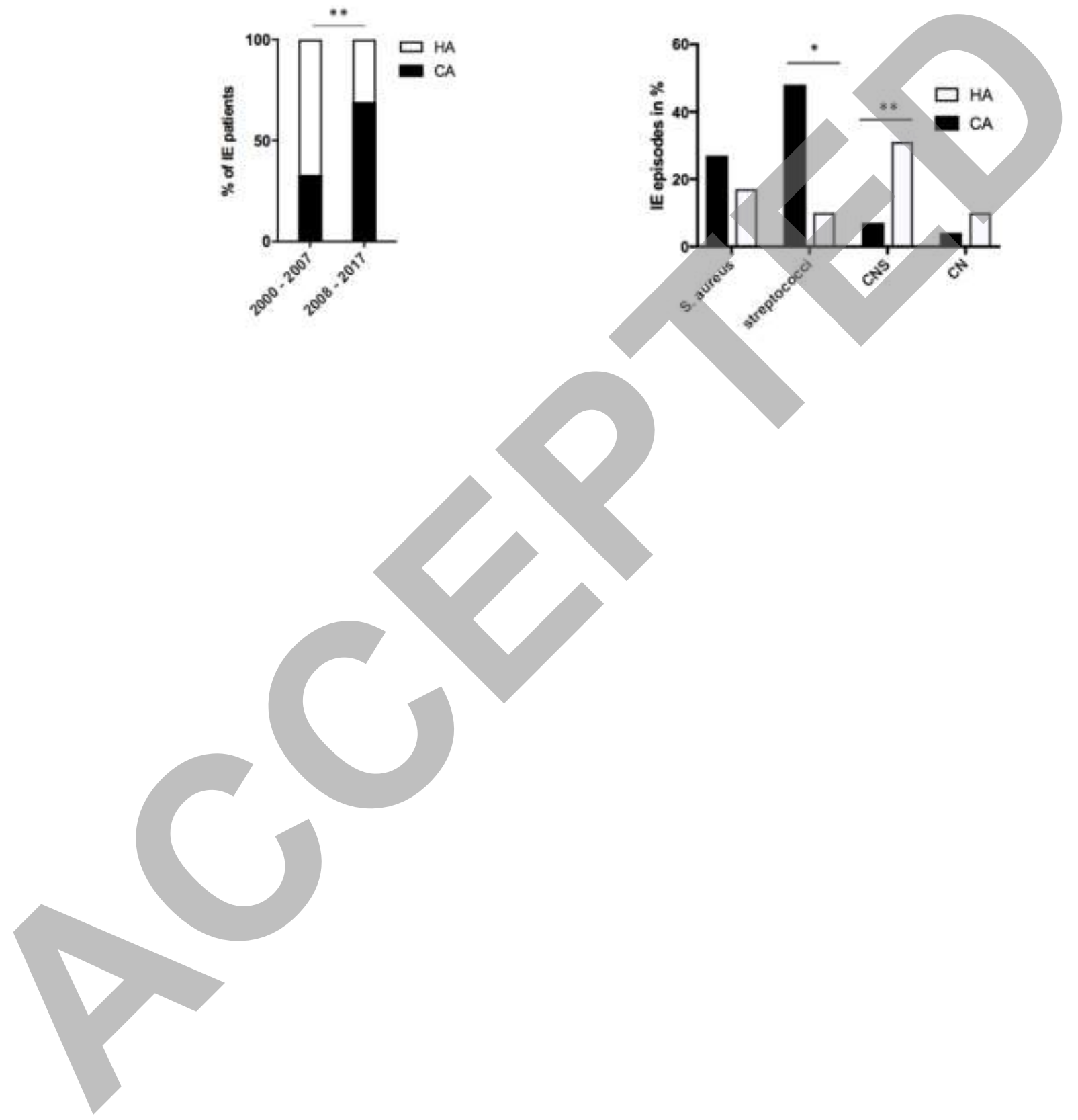

